\title{
Evidence for the effects of space weathering spectral signatures on low albedo asteroids
}

\author{
C. Lantz ${ }^{1,2}$, B. E. Clark ${ }^{3}$, M. A. Barucci ${ }^{1}$, and D. S. Lauretta ${ }^{4}$ \\ 1 LESIA - Observatoire de Paris, CNRS/UPMC (Paris 6)/Univ. Paris Diderot (Paris 7), Meudon, France \\ e-mail: cateline.lantz@obspm.fr \\ 2 UFR de Physique, Université Paris Diderot, Paris, France \\ 3 Department of Physics, Ithaca College, Ithaca (NY), USA \\ ${ }^{4}$ Lunar and Planetary Laboratory - Department of Planetary Sciences, University of Arizona, Tucson (AZ), USA
}

Received 28 March 2013 / Accepted 7 May 2013

\begin{abstract}
Context. Space weathering changes asteroid surfaces. We know from observations of the Moon and high-albedo asteroids that interplanetary surface processes can alter the spectral properties of silicates. The next step is to extend the study to primitive asteroids. This work supports the OSIRIS-REx mission by providing predictions for what space weathering effects we can expect to find on the mission target: asteroid (101955) 1999 RQ36.

Aims. To investigate the possible spectral signatures of surface processes on carbonaceous (low-albedo) asteroids, we study the reflected light spectra of carbonaceous meteorites (assumed to represent asteroid subsurface materials) and compare them with telescopic reflected light spectra of asteroids (assumed to represent asteroid surface materials).

Methods. In this work, we assume that primitive C-complex asteroids are the parent bodies of carbonaceous chondrites. We reason that differences between spectra of particulate samples of the meteorites and spectra of the regolith of asteroids can be due to either differences in textural properties, or differences caused by surface processes on the asteroid. We use telescopic observations of $\mathrm{Ch} /$ Cgh-type asteroids (0.4 to $2.4 \mu \mathrm{m}$ ) and compare them statistically with $106 \mathrm{CM}$ meteorite spectra from RELAB.

Results. Our results indicate spectral blueing of asteroids, with little to no concurrent albedo change or band modification.
\end{abstract}

Key words. minor planets, asteroids: general - meteorites, meteors, meteoroids - methods: data analysis

\section{Introduction}

The study of asteroid composition helps us to understand processes and conditions that prevailed during the first phases of inner solar system formation. Exposure of airless bodies to the harsh space environment leads to changes in their surfaces. The term space weathering includes all alteration processes such as micrometeoritic bombardment and charged particle irradiation which modify the structure and optical/chemical/mineralogical properties of the asteroid's outer layers. These phenomena degrade the accuracy of compositional interpretations made from spectra: thus it is essential to understand space weathering effects for remote sensing observations. Space weathering has been studied progressively on the various small bodies of the solar system by analogy with demonstrated effects on the Moon (Hapke 2001). Space weathering effects are not identical everywhere in the solar system; distance to the Sun, for example, is an important factor. Space weathering laboratory simulation results indicate that the grain size and the temperature of the target materials are also important (Moroz et al. 2000).

The effects of space weathering on S-type asteroids have been widely analyzed (Moroz et al. 1996; Clark et al. 2001; Brunetto et al. 2005; Ishiguro et al. 2007). Because S-types have high albedos and strong absorption band features, space weathering effects are easier to detect. Considering the fact that asteroids are parent bodies of meteorites, a good way to study space weathering spectral effects is to compare spectra of these objects: this should reveal the spectral effects of alteration processes on asteroid surfaces. Numerous laboratory experiments have been performed with meteorites in attempts to simulate the effects of space weathering. The following list summarizes the results of the main experimental approaches that have been used thus far:

- Ion irradiation is used to simulate solar wind bombardment. Experiments irradiating olivine and pyroxene all lead to general darkening, spectral reddening and mitigation of absorption bands (Brunetto et al. 2007);

- Laser irradiation is used to simulate micrometeorite impacts. Experiments with laser shots on olivine and pyroxene samples (Sasaki et al. 2002) lead to a decrease in reflectance in the visible wavelength range and spectral reddening;

- Evaporation and condensation are used to simulate impact vaporization. After a shock, vapor is created that is redeposited on the regolith with accompanying loss of oxygen. Iron reduction reactions occur resulting in the production of nanophase reduced iron (SMFe). The addition of artificial $\mathrm{SMFe}$ in manufactured silicate samples show that the presence of SMFe is a cause of spectral reddening (Noble et al. 2007).

For all experimental methods, inadvertent metamorphism of the target materials is a big concern because thermal metamorphism may also create spectral differences (Moroz 2005; Hiroi et al. 1996), and metamorphism resulting from space weathering is not considered to be an important regolith process on actual asteroid surfaces.

Three major space agencies are currently developing spacecraft missions (OSIRIS-REx for NASA, Hayabusa-2 for JAXA 
and MarcoPolo-R in assessment study at ESA) that will visit primitive low albedo asteroids that may be rich in organic compounds. Thus, there is an urgent need for an accurate model of space weathering effects on low albedo asteroids. The goal of this work is to develop a model for predicting space weathering effects on asteroid 1999 RQ36 to support the sample site selection science goals of the OSIRIS-REx mission. The target of OSIRIS-REx is a B-type asteroid that is generally considered to be related to carbonaceous chondrite meteorites. However, some controversy exists about exactly which of the carbonaceous meteorite classes is an appropriate analog for RQ36 (Clark et al. 2010; Clark et al. 2011; DeLeon et al. 2012). Therefore we have chosen to work on a related primitive asteroid type, the $\mathrm{Ch} / \mathrm{Cgh}-$ type, for which a link with CM meteorites has, by broad consensus, been established in the literature (Hiroi et al. 1996; Burbine 1998). The link is based on two quantities that distinguish these objects from other meteorites and asteroids: they both possess an absorption feature at $0.7 \mu \mathrm{m}\left(\mathrm{Fe}^{2+}-\mathrm{Fe}^{3+}\right.$ transition) (Vilas 1994), and they both have low albedos. We reason that differences between spectra of particulate samples of the meteorites and spectra of the regolith of asteroids can be due to either differences in textural properties, or differences caused by surface processes on the asteroid but are most likely not due to mineralogical differences. So, to investigate the possible spectral signatures of surface processes on carbonaceous (low-albedo) asteroids, we study the reflected light spectra of CM meteorites (assumed to represent asteroid subsurface materials) and compare them with telescopic reflected light spectra of $\mathrm{Ch} / \mathrm{Cgh}$ asteroids (assumed to represent asteroid surface materials).

\section{Previous work}

One reason we wonder if space weathering effects on S-type asteroids are also valid for carbonaceous objects is that optical effects on S-types are understood to be due to the creation of finely dispersed opaque minerals (Pieters et al. 2000; Clark et al. 2002) that are absorbent in the visible range and highly reflective in the infrared (this is the cause of the spectral reddening). But carbonaceous meteorites already have fine-grained opaque matrix (Cloutis et al. 2011): would space weathering effects be therefore "hidden" on low albedo bodies?

Space weathering simulations on carbonaceous materials have been performed, but contradictory results have emerged. Irradiation of asphaltite (organic mineral) with $\mathrm{Ar}^{++}$ions leads to a slight increase of reflectance and a spectral flattening (Moroz et al. 2004a): this is a blueing effect (as opposed to the reddening found on S-type asteroids). In contrast, another study found reddening effects for all types of asteroids (Lazzarin et al. 2006). Lazzarin et al. performed a statistical study and incorporated the spectra of pristine and altered carbonaceous chondrite Mighei obtained by Moroz et al. (2004b) who found reddening. A previous paper (Rivkin et al. 2002) predicted blueing by comparing reflective properties of SMFe and surfaces of S and C-type asteroids. Indeed, reduced iron particles have lower albedos than those of olivine and pyroxene contained in large quantities in S-type asteroids, and one can observe reflectance darkening mainly in the visible range (leading to reddening). Conversely, reduced iron particles have higher albedos than C-type asteroids: we should therefore expect an increase in reflectance (blueing) (Clark et al. 2010). In fact, low dose laser irradiation of the carbonaceous meteorite Allende initially results in blueing. However, after an increased dose of laser irradiation, Moroz et al. (1996) found that the spectrum reddens. Studying color variations between young and old asteroids families, Nesvorny et al. (2005) found that aging surfaces of S-type become increasingly redder and that aging surfaces of C-type asteroids become increasingly bluer.

Several previous space weathering studies deal with comparing samples of "pristine" and "altered" meteorites spectra respectively representing fresh (no space weathering effects) and altered (that experienced space weathering) surface materials. Here we directly compare spectra of asteroids with their meteoritic analogs. We also compare the asteroids with meteorite analog samples that experienced laser irradiation or heating experimental treatment. Our analysis thus seeks to constrain the validity of past laboratory simulations of space weathering.

\section{Data included in the study}

\subsection{Asteroids}

From the literature and our own observations we have assembled a collection of 43 (Table 1) spectra of $\mathrm{Ch} / \mathrm{Cgh}$ asteroids (DeMeo et al. 2009; Fornasier et al. 1999). Spectra were obtained mainly from the Small Main-Belt Asteroid Spectroscopic Survey II (SMASS), and from the observations of Bobby Bus, Sonia Fornasier and Beth Ellen Clark. 19 of our asteroids have data that cover the visible and near-infrared wavelength ranges ( 0.4 to $2.4 \mu \mathrm{m})$ and 24 of our asteroids have data in the visible wavelengths only $(0.4$ to $0.9 \mu \mathrm{m})$.

\subsection{Meteorites}

Next, we have taken all of the Brown University REflectance LABoratory (RELAB) spectra of CM meteorites (Clark et al. 2011) with a band center between 0.65 and $0.75 \mu \mathrm{m}$ showing a band Signal Strength Ratio (SSR) greater than 7. We have assembled 45 spectra of these meteorites (Fig. 1). To determine which meteorites showed the $0.7 \mu \mathrm{m}$ band, we have fit the continuum across the band (by fitting a line tangent to the spectral maxima on both sides of the band we want to measure), removed the slope of the continuum (by dividing the spectrum by the continuum), and fit polynomials of different degrees to the band until the band SSR was maximized (we tested all polynomials of order between 2 and 4). The band center is calculated as the position where the minimum of the polynomial occurs, and the band depth as the minimum in the spectral reflectance curve relative to the fitted continuum. What we call signal strength ratio (SSR) is a quantity used to specify the strength of the band. It is obtained by dividing the depth of the band by the standard deviation between the polynomial fit and the reflectance data. After some study we have found that a threshold value of 7 distinguishes between strong detections and weak signals in noisy data. In Fig. 1 we present all spectra with SSR greater than 7 (good $0.7 \mu \mathrm{m}$ band detections). Meteorites with SSR values lower than 7 were not included among our study objects. In Fig. 1, one can see a strange meteorite brighter and redder than the others (sample EET83389,15 with a grain size $<125 \mu \mathrm{m}$ ). This upper spectrum appears to be a statistical outlier (the spectrum is $4 \sigma$ away from the meteorites mean spectrum) so we decided to exclude it for the study. Moreover, these six meteorites were spectrally most neutral: Boriskino (grain size $<45 \mu \mathrm{m}$ ), ALH83100,196 $(<500 \mu \mathrm{m})$, Nogoya (63-125 $\mu \mathrm{m})$, Murchison (63-125 $\mu \mathrm{m})$, Murchison (unknown grain size) and Murchison $(100-200 \mu \mathrm{m})-$ as one can see at the bottom of the Fig. 1 plots. 
C. Lantz et al.: Evidence for the effects of space weathering spectral signatures on low albedo asteroids

Table 1. Asteroids used in this study.

\begin{tabular}{|c|c|c|c|c|}
\hline \multicolumn{2}{|c|}{ Asteroid } & Type & Data & Reference \\
\hline 13 & Egeria & $\mathrm{Ch}$ & VisNIR & 1 \\
\hline 19 & Fortuna & $\mathrm{Ch}$ & VisNIR & 1 \\
\hline 24 & Themis & $\mathrm{C}^{a}$ & Vis & 2 \\
\hline 34 & Circe & $\mathrm{Ch}$ & Vis & 1 \\
\hline 38 & Leda & $\mathrm{Cgh}$ & VisNIR & 1 \\
\hline 41 & Daphne & $\mathrm{Ch}$ & VisNIR & 1 \\
\hline 48 & Doris & $\mathrm{Ch}$ & VisNIR & 1 \\
\hline 49 & Pales & $\mathrm{Ch}$ & Vis & 1 \\
\hline 50 & Virginia & $\mathrm{Ch}$ & Vis & 1 \\
\hline 51 & Nemausa & Cgh & Vis & 1 \\
\hline 54 & Alexandra & $\mathrm{Cgh}$ & Vis & 1 \\
\hline 58 & Concordia & $\mathrm{Ch}$ & Vis & 1 \\
\hline 66 & Maja & $\mathrm{Ch}$ & VisNIR & 1 \\
\hline 70 & Panopaea & Cgh & Vis & 1 \\
\hline 78 & Diana & $\mathrm{Ch}$ & VisNIR & 1 \\
\hline 84 & Klio & $\mathrm{Ch}$ & Vis & 1 \\
\hline 104 & Klymene & $\mathrm{Ch}$ & Vis & 2 \\
\hline 105 & Artemis & $\mathrm{Ch}$ & Vis & 1 \\
\hline 106 & Dione & $\mathrm{Cgh}$ & VisNIR & 3 \\
\hline 111 & Ate & $\mathrm{Ch}$ & Vis & 1 \\
\hline 112 & Iphigenia & Cgh & VisNIR & 4 \\
\hline 130 & Elektra & $\mathrm{Ch}$ & VisNIR & 3 \\
\hline 137 & Meliboa & n....$^{a, b}$ & Vis & 2 \\
\hline 144 & Vibilia & $\mathrm{Ch}$ & VisNIR & 4 \\
\hline 145 & Adeona & $\mathrm{Ch}$ & Vis & 2 \\
\hline 146 & Lucina & $\mathrm{Ch}$ & Vis & 2 \\
\hline 200 & Dynamene & $\mathrm{Ch}$ & Vis & 2 \\
\hline 205 & Martha & $\mathrm{Ch}$ & Vis & 1 \\
\hline 211 & Isolda & $\mathrm{Ch}$ & Vis & 2 \\
\hline 238 & Hypatia & $\mathrm{Ch}$ & Vis & 2 \\
\hline 266 & Aline & $\mathrm{Ch}$ & VisNIR & 1 \\
\hline 284 & Amalia & $\mathrm{Ch}$ & VisNIR & 4 \\
\hline 345 & Tercidinia & $\mathrm{Ch}$ & VisNIR & 1 \\
\hline 410 & Chloris & $\mathrm{Ch}$ & Vis & 2 \\
\hline 444 & Gyptis & $\mathrm{C}^{a}$ & Vis & 2 \\
\hline 490 & Veritas & $\mathrm{Ch}$ & Vis & 2 \\
\hline 554 & Peraga & $\mathrm{Ch}$ & Vis & 4 \\
\hline 706 & Hirundo & Cgh & VisNIR & 1 \\
\hline 712 & Boliviana & n.. ${ }^{a, b}$ & Vis & 2 \\
\hline 776 & Berbericia & Cgh & VisNIR & 1 \\
\hline 1300 & Marcelle & Cgh & VisNIR & 1 \\
\hline 2099 & Opik & $\mathrm{Ch}$ & VisNIR & 1 \\
\hline 2378 & Pannekoek & $\mathrm{Cgh}$ & VisNIR & 1 \\
\hline
\end{tabular}

Notes. We give their taxonomic class (Bus-DeMeo taxonomy) and the kind of data we examined (visible or visible and near infrared wavelength ranges). ${ }^{(a)}$ We kept these objetcs because they show an absorption band at $0.7 \mu \mathrm{m} .{ }^{(b)}$ n.i. $=$ not identified in the Bus-DeMeo taxonomy.

References. (1) DeMeo et al. (2009); (2) Fornasier et al. (1999); (3) DeMeo et al. (2009) \& Bus; (4) Clark (this study).

\subsection{Laboratory altered meteorites}

We also studied 36 spectra of CM meteorites that were used for laboratory experiments. Most have been subjected to various degrees of irradiation and/or heating experiments in efforts to simulate space weathering surface processes: in Fig. 2, nine samples are laser-irradiated and 27 are heated. We call these the "altered" meteorite samples.

\section{Analysis methods}

To examine the spectral differences between our meteorites and the asteroid surfaces, we have simply divided each asteroid

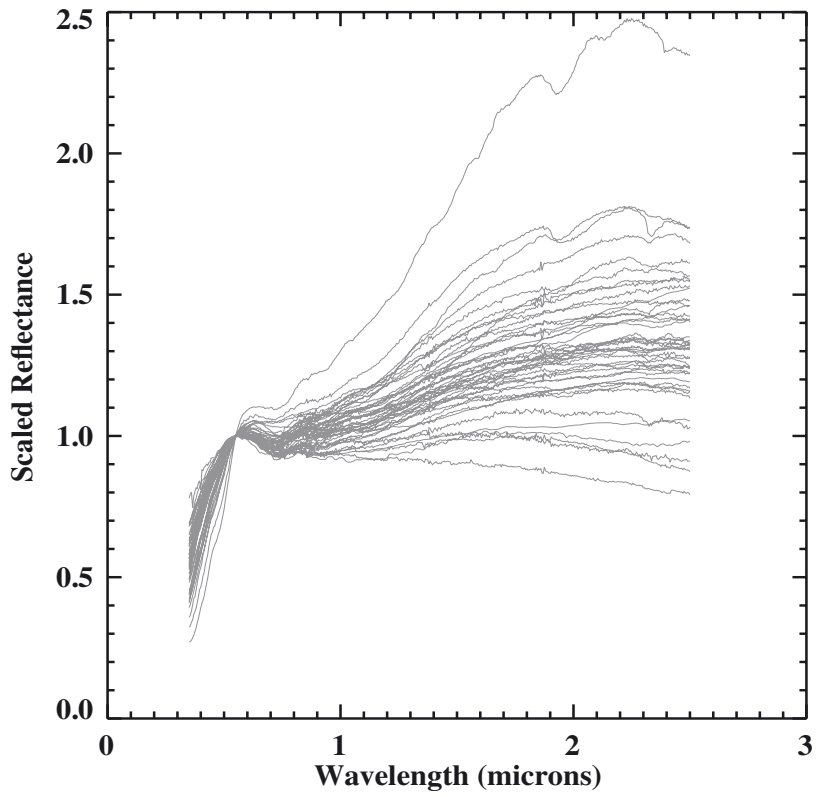

Fig. 1. Visible and near infrared spectra of CM meteorites from RELAB showing the objects showing absorption bands centered between 0.65 and $0.75 \mu \mathrm{m}$ with SSR $>7$. Data are normalized at $0.55 \mu \mathrm{m}$. The topmost spectrum is of the meteorite EET83389,15 measured at a grain size of $<125 \mu \mathrm{m}$ (RELAB file number c1mp42). This spectrum is anomalous and beyond the $2 \sigma$ variation in the rest of the dataset, so it has been eliminated for the remainder of the study.

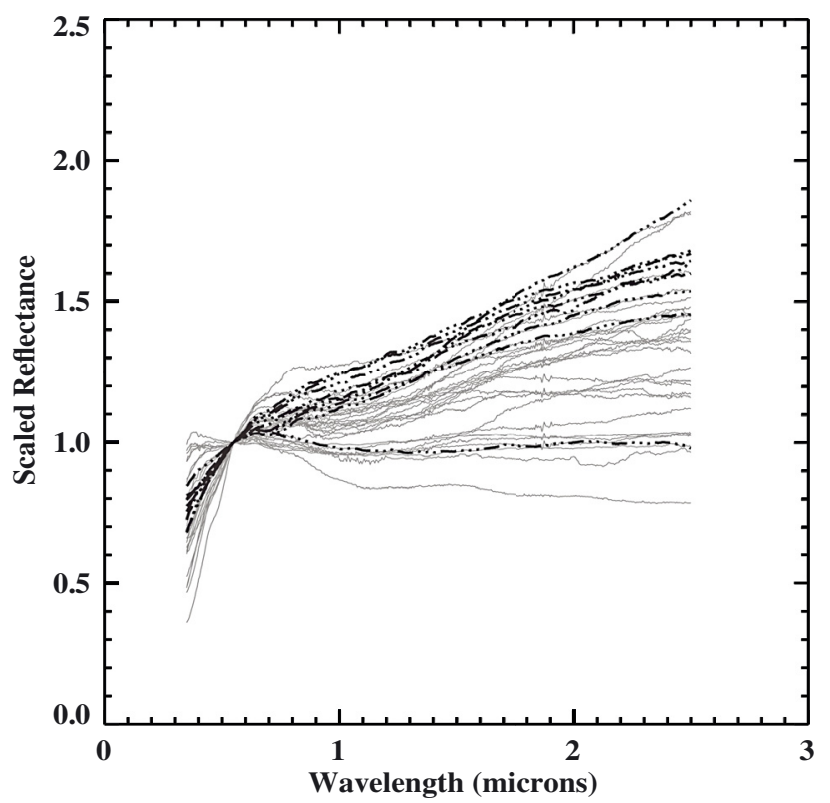

Fig. 2. Visible and near infrared spectra of laboratory altered CM meteorites from RELAB. Shown in simple lines are the heated samples, dashed/dotted lines are laser-irradiated samples. Data are normalized at $0.55 \mu \mathrm{m}$.

spectrum by each of the 44 "unaltered" meteorite spectra to produce a total of $43 \times 44=1892$ ratios. Because this is a very large number, we show only two representative examples: Fig. 3 presents combination plots of relative reflectance vs. wavelength for asteroids 13 Egeria (Ch-type) and 106 Dione (Cgh-type). Shown in gray lines are the ratios (asteroid/meteorite) for each meteorite in our data set, and shown in thick black line is an overplot of the average of the ratios. In dashed black lines are the $+\sigma$ 

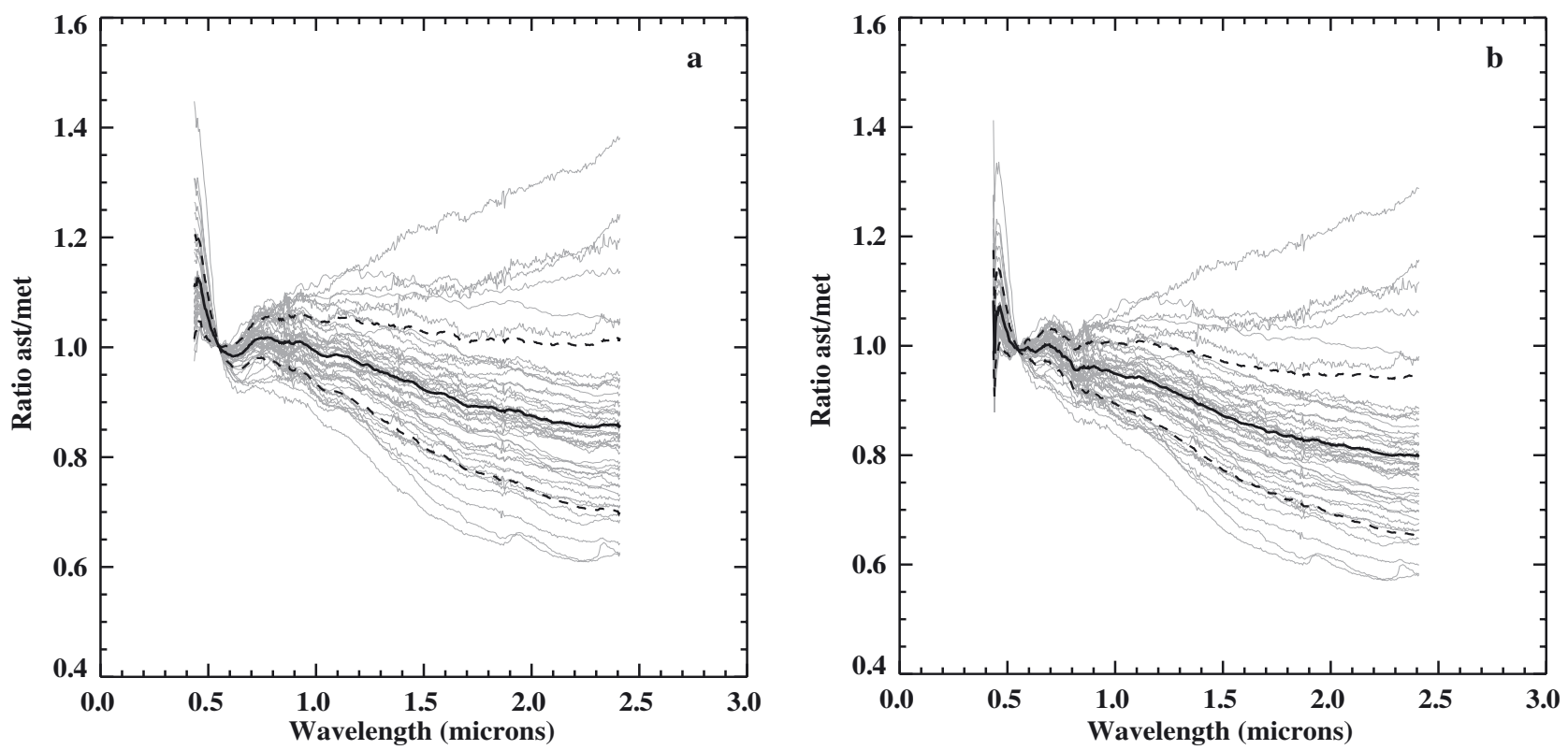

Fig. 3. Ratios for a) 13 Egeria and b) 106 Dione spectra divided by unaltered CM meteorites. Average ratios decrease with increasing wavelength, showing the spectral blueing trend we describe in the text.

and $-\sigma$ from the mean. We chose this type of ratio plot to be consistent with other studies showing the spectral effects of surface alteration on higher albedo asteroids (Clark et al. 2001). Asteroid data were smoothed before ratioing to avoid high frequency variations and focus only on low frequency behavior. We see profiles similar to Fig. 3 for all of our asteroids. The ratio is greater than 1 before the normalization point, so at lower wavelengths the asteroid reflectance is higher than meteorite reflectance. After the normalization point the ratio is smaller than 1: here the meteorites are brighter than asteroids. These two findings combined with the decrease of the ratio have led us to the conclusion that $\mathrm{Ch} / \mathrm{Cgh}$ asteroid spectra are blue compared with $\mathrm{CM}$ meteorites. We have noted that we found six meteorites with ratios consistently greater than 1 . For these six samples, a reddening effect is implied. However, for all the rest of the samples (39 of 44, $89 \%$ ) a consistent blueing effect is observed. To confirm this, we present in Fig. 4 plots of the average spectra of asteroids (thick gray line) and meteorites (thick black dashed/dotted line) with the $\pm 1 \sigma$ associated errors (thiner lines with same linestyle/color code, respectively). Because all the asteroids in our set do not have data in the NIR wavelenghts, the average for these objects has been done in the ranges $0.52-0.86$ and $0.86-2.41 \mu \mathrm{m}$. We observe clearly that the asteroids are bluer than meteorites, but we have to moderate this statement while considering the standard deviations.

Ratios allow us to quickly locate spectral differences between asteroids and meteorites, but ratios are mostly useful for seeing differences in continuum slopes and levels of reflected light. To see differences in the absorption bands, it is more useful to calculate spectral parameters. We have measured values for eight spectral parameters:

1. albedo (or brightness at $0.55 \mu \mathrm{m}$ ),

2. visible wavelength (VIS) continuum slope over the range $0.44-0.70 \mu \mathrm{m}$,

3. near-infrared wavelength (NIR1) slope over the range $1.10-1.45 \mu \mathrm{m}$,

4. near-infrared (NIR2) slope over the range 1.6-2.3 $\mu \mathrm{m}$,

5. the $0.7 \mu \mathrm{m}$ band center wavelength,

6. band center standard deviation,

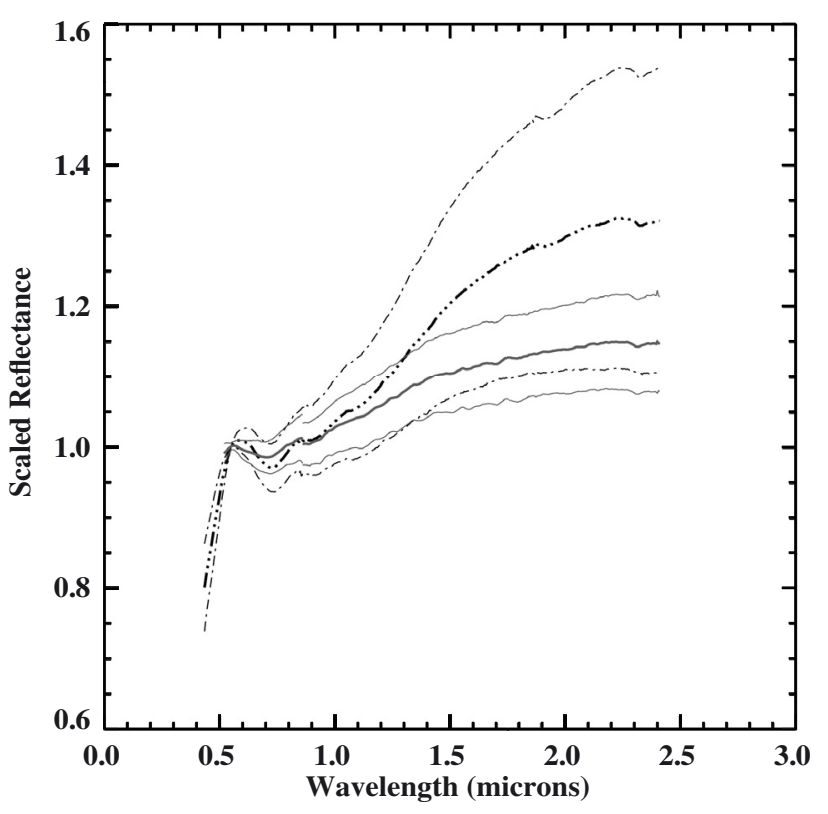

Fig. 4. Visible and near infrared average spectra of asteroids (gray) and CM laboratory-unaltered meteorites (black and dashed). The standard deviations are also shown (gray and black/dashed thiner lines, respectively). Data are normalized at $0.55 \mu \mathrm{m}$.

7. band depth,

8. band signal strength ratio (SSR, as described in the previous section).

We have measured each band three times to constrain the uncertainty in the band fit. These spectral parameters were calculated with all asteroids and meteorites (both "unaltered" and "altered") spectra. Albedo values are from IMPS data set (Tedesco et al. 2004). In some asteroid spectra, there was a second band located around $0.9 \mu \mathrm{m}$ (Cloutis et al. 2011, 2012), however we did not find this band in the meteorite spectra (including laboratory altered meteorites). Figure 5 presents plot comparisons of parameters for our study objects. Asteroids are shown in black 
C. Lantz et al.: Evidence for the effects of space weathering spectral signatures on low albedo asteroids
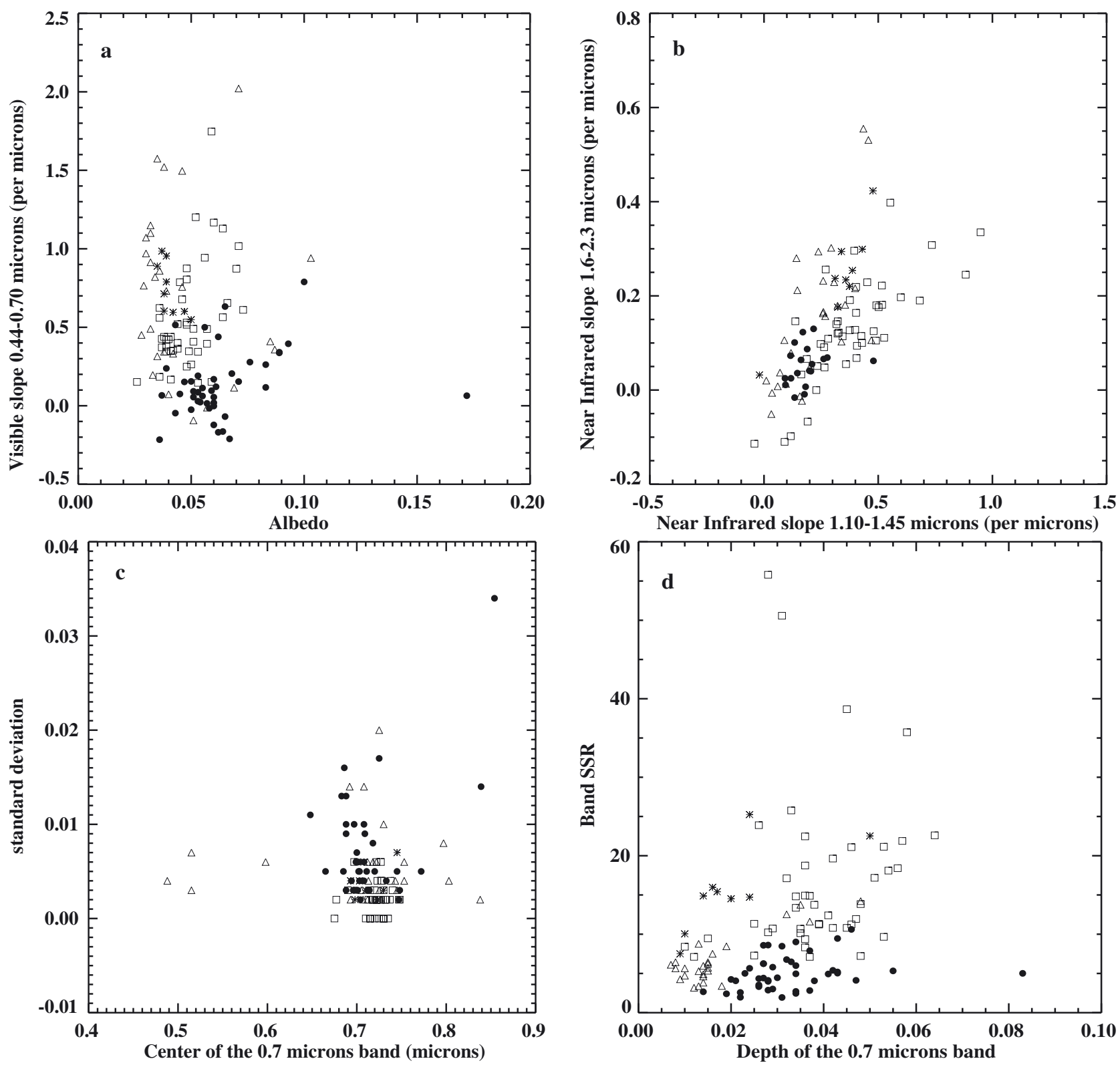

Fig. 5. Spectral parameters for asteroids (black dots), meteorites (squares) and laboratory altered meteorites (triangles for heated and stars for laser irradiated). a) Albedo vs. Vis slope $(0.44-0.70 \mu \mathrm{m})$. b) NIR1 slope $(1.10-1.45 \mu \mathrm{m})$ vs. NIR2 slope $(1.6-2.3 \mu \mathrm{m})$. c) Center of the $0.7 \mu \mathrm{m}$ band vs. standard deviation. d) Depth of the $0.7 \mu \mathrm{m}$ band vs. band SSR.

dots, meteorites are shown in squares and laboratory altered meteorites are shown in triangles and stars: heated and irradiated, respectively.

Our asteroid albedos (Fig. 5a) generally cover the range 4-10\%. The asteroid 706 Hirundo is brighter than most of our objects (17\%), but is classified as Cgh-type so we retained it in our sample. We recall, however, that the Bus-DeMeo taxonomy is based only on spectral shape and absorption bands and does not take albedo in account. Meteorites cover the range 3-8\% and laboratory altered meteorites are mainly concentrated in the $3-5 \%$ region.

In terms of the VIS slope (Fig. 5a), asteroids are mostly between -0.2 and $+0.5 \mu \mathrm{m}^{-1}$ while meteorites are mostly between 0.10 and $1.2 \mu \mathrm{m}^{-1}$. Laboratory altered meteorites cover the range $0.1-1.2 \mu \mathrm{m})$. Figure $5 \mathrm{~b}$ shows all the NIR slopes. Asteroid NIR1 slopes (recall that only half of our asteroid targets have data covering the near infrared wavelengths) are around $+0.2 \mu \mathrm{m}^{-1}$.
Meteorites go from flat to highly sloped with a maximum of $1 \mu \mathrm{m}^{-1}$. Laboratory altered meteorites cover the range from 0 to $0.5 \mu \mathrm{m}^{-1}$. Asteroids have NIR2 slopes from around 0 to around $0.15 \mu \mathrm{m}^{-1}$. Meteorites are mostly between 0 and $0.4 \mu \mathrm{m}^{-1}$, and laboratory altered meteorites cover the same range. We notice that asteroids have lower slope values than meteorites. This is consistent with the blueness of asteroid spectra as compared with the meteorite spectra.

Concerning the $0.7 \mu \mathrm{m}$ band (Figs. $5 \mathrm{c}$ and $5 \mathrm{~d}$ ), asteroids and meteorites have similar band depths and band center wavelengths but meteorites have smaller standard deviations and, hence, larger SSR than the asteroids, reflecting the lower signalto-noise of the telescopic observations. Laboratory altered meteorites have band parameters similar to the unaltered meteorites. The SSR for laboratory altered meteorite bands is very low, and accompanied with a shift toward weaker band depths (between 0.005 and 0.02 ). 
Table 2. Averages of spectral parameters for $\mathrm{Ch} / \mathrm{Cgh}$ asteroids and $\mathrm{CM}$ meteorites.

\begin{tabular}{l|c|ccc|cc}
\hline \hline & & \multicolumn{3}{|c|}{ Spectral slopes $\left(\mu \mathrm{m}^{-1}\right)$} & \multicolumn{2}{c}{$0.7 \mu \mathrm{m}$ band } \\
& \multirow{2}{*}{ Albedo (\%) } & Vis & NIR1 * & NIR2 * & Center $(\mu \mathrm{m})$ & Depth $(\%)$ \\
& & $0.44-0.70 \mu \mathrm{m}$ & $1.10-1.45 \mu \mathrm{m}$ & $1.6-2.3 \mu \mathrm{m}$ & & \\
\hline Asteroids & $6 \pm 2$ & $0.13 \pm 0.22$ & $0.19 \pm 0.09$ & $0.05 \pm 0.04$ & $0.71 \pm 0.04$ & $3 \pm 1$ \\
Meteorites & $5 \pm 1$ & $0.55 \pm 0.33$ & $0.39 \pm 0.19$ & $0.13 \pm 0.11$ & $0.72 \pm 0.01$ & $4 \pm 1$ \\
Altered meteorites & $4 \pm 2$ & $0.73 \pm 0.47$ & $0.25 \pm 0.14$ & $0.17 \pm 0.15$ & $0.68 \pm 0.20$ & $2 \pm 1$ \\
\hline
\end{tabular}

Notes. For each spectral parameter, we present the mean value and the associated standard deviation for the asteroids, the meteorites, and the altered meteorites, respectively. ${ }^{(*)}$ Recall that the NIR slopes were measured only for asteroids that have available data in this wavelength range. Calculations are presented for 43 asteroids (19 with NIR data), 44 meteorites and 36 laboratory altered meteorites.

We present in Table 2 the average of these parameters for each population of objects (asteroids, meteorites and altered meteorites).

\section{Discussion}

We recall that for meteorites, the reflectance value at $0.55 \mu \mathrm{m}$ is considered to be roughly equivalent to the geometrical albedo of an asteroid, even if it is difficult to compare quantities between asteroids and meteorites (Barucci et al. 2012). The lack of a difference between the asteroid albedos and the meteorite brightnesses (Fig. 5a) could be due to the fact that surface processes have no effect on carbonaceous asteroid albedo. Indeed, strong albedo effects were not expected with carbonaceous materials because of their opaque mineral-rich compositions (Pieters et al. 2000). There is a marked decrease in the albedo of laboratory altered meteorites compared with unaltered meteorites and asteroids. This could indicate that laboratory alteration experiments have not yet effectively simulated asteroid surface processing or space weathering effects.

We also notice a decrease of the VIS slope parameters for the asteroids compared with the meteorites, but no real effects for laboratory altered meteorites (Fig. 5a). This indicates that we need more laboratory alteration experiments to reproduce the spectral variations observed on asteroids in order to simulate space weathering. There is a good correlation between the NIR slopes of the asteroids and the meteorites (Fig. 5b). One can see that asteroids have lower slope parameter values than meteorites. This, together with the observed decrease of the VIS slope, indicates that asteroids are spectrally bluer than the meteorites. Laboratory altered meteorites cover the entire range in parameter values occupied by the meteorites and asteroids. It is thus possible that some experiments have effectively simulated asteroid surface spectra and some have not: a detailed investigation of what processes induce spectral slope modification is needed (mineralogy, grain size, temperature effects, etc.).

We would have liked to search for differences in the $0.7 \mu \mathrm{m}$ band between the asteroids and the meteorites (Figs. 5c and 5d). However, because we selected our study objects based on the $0.7 \mu \mathrm{m}$ band intensity, there is a bias in our sample that precludes any conclusions about differences in the band depth. We selected only meteorites with SSR at the $0.7 \mu \mathrm{m}$ band of 7, and DeMeo et al. (2009) selected only asteroids with a "detectable" $0.7 \mu \mathrm{m}$ band to form the Cgh and Ch-types of the taxonomy.

\section{Conclusion}

We have investigated the possible spectral effects of surface alteration processes on low-albedo asteroids by comparing
43 primitive asteroid spectra (in the visible and near-infrared wavelength ranges) with spectra of 44 meteorites that are derived from low-albedo asteroids. Our hypothesis is that $\mathrm{Ch} / \mathrm{Cgh}$ asteroids and carbonaceous chondrites (CM meteorites) are the same material, but the $\mathrm{Ch} / \mathrm{Cgh}$ asteroids represent airless body surfaces exposed to possible alteration by space weathering and the CM meteorites represent the fresh subsurface material. Systematic differences between these asteroid and meteorite spectra may be due to one or more of the following causes: systematic error of measurement; consistent textural differences; and/or space weathering of the asteroid surface. In this paper, we favor the space weathering explanation, and we quantify possible associated spectral signatures of space weathering on $\mathrm{CM}$ meteorite material. Our findings indicate a blueing effect of $\mathrm{Ch} / \mathrm{Cgh}$ asteroid spectra as compared with $\mathrm{CM}$ meteorites. We find a decrease in the reflectance toward long wavelengths and a neutralization of the spectral slopes. This effect is strongest in the visible range. Our results are opposite the reddening effect found by Lazzarin et al. (2006) and Moroz et al. (2004b), but consistent with the blueing effect inferred by Nesvorny et al. (2005) and Moroz et al. (2004a). Our results are opposite the well-known reddening trend found (by numerous workers) to occur on S-type asteroids (e.g. Pieters et al. 2000; Hapke 2001; Clark et al. 2002; Sasaki et al. 2001, 2002; Ishiguro et al. 2007).

Very few space weathering studies have been conducted that are relevant to primitive asteroids and they have all produced different conclusions. In particular, a study by Moroz et al. (1996) showed that weak irradiation of a carbonaceous chondrite led to a blueing of the spectrum. With higher irradiation doses, they found a reddening. It may ultimately be possible to obtain an age of the asteroid surface using a timescale of space weathering effects based on laboratory simulations (Sasaki et al. 2001). Until an asteroid surface sample is returned, we will not know how accurate the laboratory experiment techniques are at simulating the effects of space weathering. Future efforts in laboratory simulations should focus on carbonaceous target material and include mineralogy, grain size and temperature effects.

With our method of measuring spectral parameters, we are not sensitive to transformations in chemical compounds or physical properties that may also occur with space weathering. However, we are planning new laboratory experiments with different doses of irradiation on carbonaceous chondrites and we plan to study how the experiments may affect asteroid color trends (e.g. Kanuchova et al. 2012).

Acknowledgements. We thank Bobby Bus, Francesca DeMeo and Sonia Fornasier for sharing data, and Rick Binzel, Rosario Brunetto, Ed Cloutis, Marcello Fulchignoni and Maureen Ockert-Bell for useful discussions. We also thank the anonymous referee for her/his review. C.L. is grateful to the Physics department of Ithaca College for hosting a three-month visit. 
C. Lantz et al.: Evidence for the effects of space weathering spectral signatures on low albedo asteroids

\section{References}

Barucci, M. A., Belskaya, I. N., Fornasier, S., et al. 2012, Planet. Space Sci., 66, 23

Brunetto, R., \& Strazzulla, G. 2005, Icarus, 179, 265

Brunetto, R., Roush, T. L., Marra, A. C., \& Orofino, V. 2007, Icarus, 191, 381 Burbine, T. H. 1998, M\&PS, 33, 253

Clark, B. E., Lucey, P., Helfenstein, P., et al. 2001, M\&PS, 36, 1617

Clark, B. E., Hapke, B., Pieters, C. M., \& Britt, D. 2002, in Asteroids III, eds. W. F. Bottke Jr, A. Cellino, P. Paolicchi, \& R. R. Binzel (Tucson: Univ. of Arizona Press), 585

Clark, B. E., Ziffer, J., Nesvorny, D., et al. 2010, J. Geophys. Res., 115

Clark, B. E., Binzel, R. P., Howell, E. S., et al. 2011, Icarus, 216, 462

Cloutis, E. A., Hudon, P., Hiroi, T., Gaffey, M. J., \& Mann, P. 2011, Icarus, 216, 309

Cloutis, E. A., Hudon, P., Hiroi, T., \& Gaffey, M. J. 2012, Icarus, 220, 586

DeLeon, F. E., Pinilla-Alonso, N., Licandro, J., Campins, H., \& Marzo, G. A. 2012, Icarus, 218, 196

DeMeo, F. E., Fornasier, S., Barucci, M. A., et al. 2009, Icarus, 202, 160

Fornasier, S., Lazzarin, M., Barbieri, C., \& Barucci, M. A. 1999, A\&AS, 135, 65

Hapke, B. 2001, J. Geophys. Res., 106, 10039
Hiroi, T., Zolensky, M. E., Pieters, C. M., \& Lipschutz M. E. 1996, M\&PS, 31, 321

Ishiguro, M., Hiroi, T., Tholen, D. J., et al. 2007, M\&PS, 42, 1791

Kanuchova, Z., Brunetto, R., Melita, M., \& Strazzulla, G. 2012, Icarus, 221, 12 Lazzarin, M., Marchi, S., Moroz, L. V., et al. 2006, ApJ, 647, 179

Moroz, L. V. 2005, LPSC, 36, 2056

Moroz, L. V., Fisenko, A. V., Semjonova, L. F., Pieters, C. M., \& Korotaeva, N. N. 1996, Icarus, 122, 366

Moroz, L. V., Schade, U., \& Wäsch, R. 2000, Icarus, 147, 79

Moroz, L. V., Baratta, G., Strazulla, G., et al. 2004a, Icarus, 170, 214

Moroz, L. V., Hiroi, T., Shingareva, T. V., et al., 2004b, LPSC 35, 1279

Nesvorny, D., Jedicke, R., Whiteley, R. J., \& Ivezic, Z. 2005, Icarus, 173, 132

Noble, S., Pieters, C. M., \& Keller, L. P. 2007, Icarus, 192, 629

Pieters, C. M., Taylor, L. A., Noble, S., et al. 2000, M\&PS, 35, 1101

Rivkin, A. S., Howell, E. S., Vilas, F., \& Lebofsky, L. A. 2002, in Asteroids III, eds. W. F. Bottke Jr, A. Cellino, P. Paolicchi, \& R. R. Binzel (Tucson: Univ. of Arizona Press), 235

Sasaki, S., Nakmura, K., Hamabe, Y., Kurahashi, E., \& Hiroi, T. 2001, Nature, 410,555

Sasaki, S., Hiroi, T., Nakamura, K., et al. 2002, AdSpR, 29, 783

Tedesco, E. F., Noah, P. V., Noah, M., \& Price, S. D., 2004, IRAS Minor Planet Survey, IRAS-A-FPA-3-RDR-IMPS-V6.0. NASA Planetary Data System

Vilas, F. 1994, Icarus, 111, 456 